\title{
XMM-Newton observations of four high-z quasars
}

\author{
E. Ferrero ${ }^{1}$ and W. Brinkmann ${ }^{2}$ \\ 1 Max-Planck-Institut für extraterrestrische Physik, Postfach 1312, 85741 Garching, Germany \\ 2 Centre for Interdisciplinary Plasma Science, Max-Planck-Institut für extraterrestrische Physik, Postfach 1312, \\ 85741 Garching, Germany
}

Received 7 November 2002 / Accepted 18 February 2003

\begin{abstract}
We present the results of XMM-Newton observations of four high- $z$ quasars, two radio-loud and two radio-quiet. One of the radio-loud objects, PKS 2126-158, clearly shows absorption in excess of the galactic value as claimed before from ASCA and ROSAT observations. For PKS 2149-306 the evidence for excess absorption is only marginal in contrast to previous results. The location of the absorber in PKS 2126-158 is compatible with the redshift of the source. Both, a warm and a cold absorber are allowed by the X-ray data. Both quasars have very flat photon spectra $(\Gamma \lesssim 1.5)$ and the high photon statistics reveal small deviations from a simple power law form. For the two radio-quiet objects, Q 0000-263 and Q 1442+2931, we determine, for the first time, reliable spectral parameters. Both quasars have steeper power laws $(\Gamma \sim 2)$ and show absorption consistent with the galactic value, similar to radio-quiet quasars at low redshifts. In the case of Q 0000-263 the presence of the damped Ly $\alpha$ system $\left(N(\mathrm{HI}) \sim 2.6 \times 10^{21} \mathrm{~cm}^{-2}\right.$ at $\left.z=3.39\right)$ cannot be examined further due to the limited photon statistics in all instruments.
\end{abstract}

Key words. galaxies: active - quasars: general - X-rays: galaxies

\section{Introduction}

High redshift quasars are the most luminous and most distant, continuously emitting sources of electromagnetic radiation in the observable Universe. In particular at X-ray energies luminosities greater than $10^{47} \mathrm{erg} \mathrm{s}^{-1}$ are observed for some objects (Brinkmann et al. 1995). These extreme luminosities imply the presence of super-massive black holes $\left(M_{\mathrm{BH}} \sim 10^{9} M_{\odot}\right)$ in the centers of the sources and therefore provide severe constraints on theories of structure formation in the early Universe. High redshift quasars are key objects to understand the cosmological evolution of the physical properties in and around quasars on the longest possible time scales and they present rare, directly observable tracers of the physical conditions of the early Universe.

The X-ray band is important for at least two reasons: the $\mathrm{X}$-ray luminosity represents a large fraction of the bolometric luminosity of quasars (Elvis et al. 1994a) and it is known from variability and spectral studies (e.g. Mushotzky et al. 1993; Yuan et al. 1998a; Yuan \& Brinkmann 1998) that the X-rays are emitted very close to the central engine - a region which is not accessible at any other wavelength with current instrumentation.

To understand quasars a detailed knowledge is required about the mechanisms of the quasar emission and about the cosmical evolution of the objects. Radio, optical, and X-ray luminosity functions indicate that the typical luminosity of a

Send offprint requests to: E. Ferrero, e-mail: ferrero@mpe.mpg.de quasar at $z \sim 3$ is higher by a factor of $50-100$ compared to a local one (Boyle et al. 1993), but the result is dominated by a few high redshift quasars with extreme luminosities. How are these enormous amounts of radiative energy produced and do these properties evolve with time? What is the origin of the radio-loud / radio-quiet dichotomy, i.e., the fact that about $10 \%$ of the quasars show significant radio emission, are brighter at high energies and more variable?

Early studies of low- $z$ quasars by the Einstein observatory (Zamorani et al. 1981; Wilkes \& Elvis 1987) and ROSAT (Brinkmann et al. 1997; Yuan et al. 1998a) revealed X-ray luminosities up to $\sim 10^{47} \mathrm{erg} \mathrm{s}^{-1}$, which are roughly proportional to the corresponding optical luminosities, with a large dispersion. The X-ray spectra in the soft band can be described by power laws with a wide range of slopes with averages around $\Gamma \gtrsim 1.5$ for radio-loud quasars and $\Gamma \gtrsim 2.0$ for radio-quiet quasars, both flattening with increasing redshift. In the harder ASCA energy band radio-loud quasars have approximately $\Gamma \sim 1.6$ and radio-quiet quasars $\Gamma \sim 1.9$ (Reeves \& Turner 2000). At a given optical luminosity radio-loud quasars are typically $\sim 3$ times more $\mathrm{X}$-ray luminous than radio-quiet quasars. A measure for this luminosity ratio is the X-ray loudness $\alpha_{\mathrm{ox}}$, the broad spectral index of a nominal power law from the optical to the X-ray band. From Einstein and ROSAT observations (Zamorani et al. 1981; Wilkes \& Elvis 1987; Brinkmann et al. 1997; Yuan et al. 1998a) it is seen that this quantity is smaller for radio-loud quasars $\left(\alpha_{\mathrm{ox}, \mathrm{rl}} \sim 1.25\right)$ than for radio-quiet quasars $\left(\alpha_{\mathrm{ox}, \mathrm{rq}} \sim 1.6\right)$. The observationally found dependence 
of $\alpha_{\mathrm{ox}}$ on redshift and optical luminosity, where the primary correlation is that on optical luminosity (Avni \& Tananbaum 1982, 1986; Wilkes et al. 1994; Yuan et al. 1998a), would imply a different evolution in the optical and X-ray regime. However, Brinkmann et al. (1997) and Yuan et al. (1998b) argue that this dependence is not a physical property of the population but can be introduced by selection effects and the luminosity dispersions of the samples in the optical and X-ray band.

The differences between the two classes of quasars can be explained in a two component emission model (Zamorani et al. 1981; Wilkes \& Elvis 1987), where a steep soft component, linked to the optical emission, is present in all quasars and a second flat spectrum hard component linked to the radio emission through the SSC mechanism dominates the X-ray emission of radio-loud quasars.

The question of whether quasars do exhibit spectral evolution or not is fundamental and has direct impact on quasar formation models. The question of whether the observed difference between radio-quiet and radio-loud quasars at low redshifts persists to high redshifts cannot be answered conclusively with current data, which tentatively indicate that no evolution takes place in radio-loud quasars, but the number of objects is still very low. Spectra for high redshift radio-quiet quasars with reasonable quality hardly exist. A study of the currently unknown spectral properties of high redshift radio-quiet quasars is also relevant for our understanding of the cosmic $\mathrm{X}$-ray background and the contribution of these objects.

One of the major results of the ROSAT and ASCA observations of high redshift radio-loud quasars was the detection of absorption in excess of what is expected from the galactic $N_{\mathrm{H}}$-value (Elvis et al. 1994b; Siebert et al. 1996; Brinkmann et al. 1997; Cappi et al. 1997; Yuan et al. 2000). However, due to the low signal-to-noise of the spectra and the insufficient energy resolution of the instruments, it is impossible to unambiguously determine whether the absorption is galactic, inter galactic or intrinsic to the quasar and, in some cases, the absorption appears to be even temporarily variable (Schartel et al. 1997). Current observations tentatively indicate an intrinsic absorption site. Related to this is the question, whether excess absorption is also a feature of radio-quiet quasars (Yuan et al. 1998a; Yuan \& Brinkmann 1998). Up to now, the answer is no, but the data are far from being conclusive. Any systematic differences in the spectral and/or absorption properties between radio-loud and radio-quiet quasars can be extremely important for an understanding of the formation processes for quasars and the radio-loud/radio-quiet dichotomy.

Furthermore, if damped Ly $\alpha$ systems are in the line-ofsight, it should be possible, with the help of X-ray observations, to determine the ionization state of these systems and thus derive limits on the size, temperature and density for them (Fang \& Canizares 2000). However, so far current data do not give significant constraints (Fang et al. 2001).

In this paper we present the results of XMM-Newton observations of four high redshift quasars. Two are radio-loud, PKS 2126-158 $(z=3.27)$ and PKS $2149-306(z=2.34)$ and two are radio-quiet, Q $1442+2931(z=2.64)$ and Q 0000-263 $(z=4.10)$. All of them have been observed in X-rays before. From ASCA data (as well as ROSAT data in the case of
PKS 2126-158) Cappi et al. (1997) claim excess absorption towards the radio-loud objects. No excess absorption towards the radio-quiet objects was found from ROSAT observations (Bechtold et al. 1994a; Reimers et al. 1995; Kaspi et al. 2000).

Q 0000-263 was observed by ROSAT in a PSPC pointed observation on November 30, 1991. From this observation Bechtold et al. (1994a) found an energy index $\alpha=1.30 \pm 0.23$ and no indications for extra absorption. From this observation and from a second PSPC pointing on November 26, 1991 Kaspi et al. (2000) determined an unabsorbed flux in the $0.1-2.0 \mathrm{keV}$ band of $f_{0.1-2.0 \mathrm{keV}}=6.5 \times 10^{-14} \mathrm{erg} \mathrm{cm}^{-2} \mathrm{~s}^{-1}$ and an optical to $-\mathrm{X}$-ray index $\alpha_{\mathrm{ox}}=1.65$. However, they had to assume a photon index $\Gamma=2.0$ and galactic absorption as the data were insufficient to perform a spectral fit.

The discovery of Q 1442+2931 was reported by Sanduleak \& Pesch (1989) and the source was first observed in X-rays by the ROSAT PSPC in November 1992 and in July 1993 (Reimers et al. 1995). The accumulated net counts were not sufficient to allow a spectral analysis, however Reimers et al. (1995) find no indications for excess absorption as the photons were distributed over the whole ROSAT energy band.

PKS 2126-158 was detected in X-rays by Einstein (Zamorani et al. 1981). The ROSAT PSPC spectrum was presented in Elvis et al. (1994b) and from ASCA observations Serlemitsos et al. (1994) constrained the redshift of the absorber at $z<0.4$. However, Cappi et al. (1997) could not reproduce these results and attribute them to the use of older response matrices by Serlemitsos et al. (1994).

With a 2-10 keV X-ray luminosity of $L_{\mathrm{x}} \sim 6 \times 10^{47} \mathrm{erg} \mathrm{s}^{-1}$ PKS 2149-306 is one of the most luminous radio-loud quasars in the Universe. The ROSAT All Sky Survey data and the ASCA observations of PKS 2149-306 were first discussed in Siebert et al. (1996). The absorption column density found by Cappi et al. (1997) is slightly higher, but consistent, with that given by Siebert et al. (1996). However, the extra absorption $\left(\Delta N_{\mathrm{H}} \sim 4 \times 10^{20} \mathrm{~cm}^{-2}\right)$ is not large and the deduced value could be affected significantly by calibration uncertainties of the SIS detectors.

The 2-10 keV flux of $8 \times 10^{-12} \mathrm{erg} \mathrm{cm}^{-2} \mathrm{~s}^{-1}$ reported from a BeppoSAX observation in October 1997 (Elvis et al. 2000) is only $\sim 80 \%$ of the ASCA flux in 1994 (Cappi et al. 1997). The hard power law index of $\Gamma=1.4 \pm 0.05$ is similar to the values found by ASCA ( $\Gamma=1.54 \pm 0.05$, Cappi et al. 1997), however, the LECS showed an excess of counts below $1 \mathrm{keV}$ and the absorption had to be fixed to the galactic value. No evidence for a red-shifted $\mathrm{Fe}-\mathrm{K}$ emission line was seen in the spectrum and an upper limit of $63 \mathrm{eV}$ for the equivalent width of a line at $\sim 5 \mathrm{keV}$ claimed by Yaqoob et al. (1999) was given.

In recent Chandra observations Fang et al. (2001) do not find significant excess absorption towards PKS 2149-306. The source flux has decreased by about $30 \%$ compared to the ASCA observation and the deduced power law index $\Gamma=1.255 \pm 0.020$ is significantly lower than the value of $\Gamma \sim 1.55$ seen by ASCA. Further, the emission feature around $5 \mathrm{keV}$ reported by Yaqoob et al. (1999) was not found in the Chandra data.

In this paper we will present the results of XMM-Newton observations of these four quasars. In the next section we will give details on the observations and discuss the temporal 


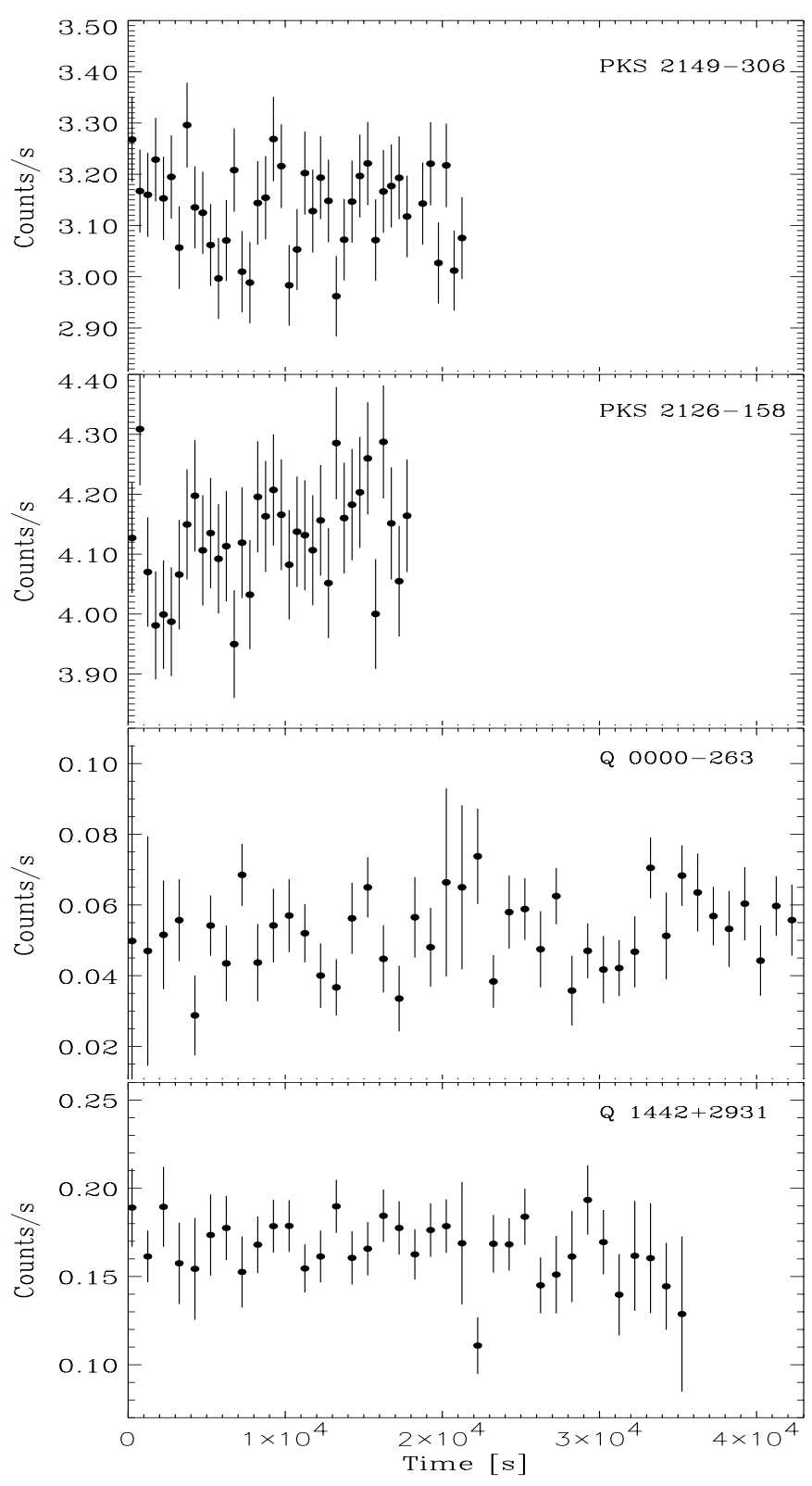

Fig. 1. Combined PN+MOS, background subtracted, 0.2-10 keV lightcurves of the four quasars. The time binning is $500 \mathrm{~s}$ for the radioloud quasars and $1000 \mathrm{~s}$ for the radio-quiet quasars.

behavior of the objects. We will then present the spectral analyses of the objects and discuss in Sect. 4 their broad band properties, in particular the amount of absorption towards the sources. A general discussion and a summary will be given in Sect. 5 .

\section{The data}

The observational details for the four sources are reported in Table 1. All PN and MOS data were reprocessed using XMMSAS version 5.3.0; for the RGS data XMMSAS version 5.3.3 has been used.

\subsection{The light curves}

We calculated the $0.2-10 \mathrm{keV}$ light curves for all four objects by extracting the photons from a circular region centered on the source with a radius of $45^{\prime \prime}$. This extraction radius was chosen to avoid contamination from nearby objects and it contains about $90 \%$ of the source photons, using the encircled energy function given by Ghizzardi \& Molendi (2001). Only single and double events (i.e. with pattern 0-4 for the PN camera and 0-12 for the MOS cameras) and with quality flag 0 were chosen. The time bin size was set to be $500 \mathrm{~s}$ for the radio-loud objects and to $1000 \mathrm{~s}$ for the radio-quiet ones and we used only the time range for which all EPIC cameras were switched on.

The backgrounds were determined with the same selection criteria from source free regions on the same chips and subtracted from the source light curves. The co - added PN and MOS net light curves for the four objects are shown in Fig. 1.

The light curve of PKS 2149-306 is consistent with a constant average flux during the observation period. A Kolmogorov-Smirnov test gives a $\leq 15 \%$ probability that the short time variability is only that expected from purely statistical fluctuations. However, no definite time scale can be deduced from the relatively noisy data.

PKS 2126-158 shows a slight increase of the count rate with time of $\sim(4.8 \pm 2.7) \times 10^{-6} \mathrm{cts} \mathrm{s}^{-2}$ as well as short time flux variations of $\sim 5 \%$ on time scales of $\sim 1 \mathrm{ksec}$. These variations seem to occur in a well organized linear fashion but the counting statistics are insufficient for a more detailed analysis. In both cases these variations are moderate and not unusual amongst radio loud quasars. From the observed variability we can estimate a lower limit to the radiative efficiency $\eta \gtrsim 5 \times 10^{-43} \Delta L / \Delta t$ (Fabian 1979). With the above given time scale and the luminosity determined from the spectral analysis (see Sect. 3) we obtain $\eta \gtrsim 8$. This value largely exceeds the theoretical limit of accretion onto a black hole implying enhancement of the emission by relativistic beaming.

The light curves of the two radio-quiet objects Q 0000-263 and Q 1442+2931 are consistent with a constant flux and show no statistically significant variations. The drop of the count rate at the end of the Q 1442+2931 observation and the one exceptional low point coincide with strong flaring activity of the background and are thus very likely not source intrinsic intensity variations.

\section{Spectral analysis}

We have performed the spectral analysis of PN, MOS and RGS data for both radio-loud quasars using the latest available versions of the response matrices, released in April 2002 (PN) and in March 2002 (MOS). The RGS response matrices were created with the SAS package rgsrmfgen. In the case of the radioquiet quasars we have analyzed only the PN and MOS data, because of the low photon statistics in the RGS spectra. As a preliminary step, we have created Good Time Interval (GTI) files to check for time intervals with high background which could contaminate significantly the source photons. For both the radio-loud sources the background remained low during 
Table 1. Data of observations.

\begin{tabular}{|c|c|c|c|c|c|}
\hline Source & Observing date & Instrument & Mode & Filter & $\begin{array}{c}\text { Exposure } \\
(\mathrm{ksec})\end{array}$ \\
\hline PKS 2149-306 & May 1, 2001 & $\begin{array}{c}\text { PN } \\
\text { MOS } 1 / 2 \\
\text { RGS1/2 } \\
\text { OM }\end{array}$ & $\begin{array}{l}\text { Large Window } \\
\text { Full Window } \\
\text { Spectroscopy HER } \\
\text { Imaging }\end{array}$ & $\begin{array}{l}\text { Medium } \\
\text { Medium }\end{array}$ & $\begin{array}{l}\sim 21.8 \\
\sim 24 \\
\sim 25 \\
\sim 20^{\dagger}\end{array}$ \\
\hline PKS 2126-158 & May 1, 2001 & $\begin{array}{l}\mathrm{PN} \\
\mathrm{MOS} 1 / 2 \\
\mathrm{RGS} 1 / 2 \\
\mathrm{OM}\end{array}$ & $\begin{array}{l}\text { Extended Full Window } \\
\text { Full Window } \\
\text { Spectroscopy HER } \\
\text { Imaging }\end{array}$ & $\begin{array}{l}\text { Medium } \\
\text { Medium } \\
\text { Grism } 1\end{array}$ & $\begin{array}{l}\sim 18 \\
\sim 22.8 \\
\sim 23 \\
\sim 20^{\dagger}\end{array}$ \\
\hline Q 0000-263 & June 25, 2002 & $\begin{array}{c}\mathrm{PN} \\
\mathrm{MOS} 1 / 2 \\
\mathrm{RGS} 1 / 2\end{array}$ & $\begin{array}{l}\text { Extended Full Window } \\
\text { Full Window } \\
\text { Spectroscopy HER }\end{array}$ & $\begin{array}{l}\text { Thin } \\
\text { Thin }\end{array}$ & $\begin{array}{c}\sim 43.5 \\
\sim 50.3 \\
\sim 23\end{array}$ \\
\hline Q $1442+2931$ & August 1, 2002 & $\begin{array}{c}\mathrm{PN} \\
\mathrm{MOS} 1 / 2 \\
\mathrm{RGS} 1 / 2 \\
\mathrm{OM}\end{array}$ & $\begin{array}{l}\text { Extended Full Window } \\
\text { Full Window } \\
\text { Spectroscopy HER } \\
\text { Imaging }\end{array}$ & $\begin{array}{l}\text { Thin } \\
\text { Thin } \\
\text { UVW2 }\end{array}$ & $\begin{array}{l}\sim 37 \\
\sim 39 \\
\sim 42 \\
\sim 20^{\dagger}\end{array}$ \\
\hline
\end{tabular}

$\dagger$ Divided into five exposures of $\sim 4 \mathrm{ksec}$ each.

the whole observation, so that no time intervals had to be excluded from the subsequent analysis. During the observations of the two radio-quiet objects several background flares were found and the flaring time intervals were excluded in the spectral analysis.

We also checked for pile up exploiting the XMMSAS task epatplot, both for the PN and the MOS cameras. We found no indications for it in the radio-quiet sources and in PKS 2149-306, whereas for PKS 2126-158 signs for pile up are present for the PN camera with a count rate of $\sim 2.7 \mathrm{cts} / \mathrm{s}$ which is close to the critical value for a point source in the Extended Full Window mode. No pile up was found for the MOS cameras.

For the PN camera we extracted the photons from a circular region of radius $45^{\prime \prime}$ centered on the X-ray positions of all four quasars. This extraction radius corresponds to the maximum allowed to avoid the chip boundaries. In the case of PKS 2126-158 we also excluded from the analysis the four central (RAW-) pixels in order to avoid pile up. The same extraction radius as for the PN was used for the MOS cameras. The backgrounds were extracted from source free regions with the same radius from positions near the source. Only single and double events with flag 0 were selected for the PN, whereas only photons with pattern 0-12 and flag 0 were chosen for the MOS (for details on the XMM detectors see Ehle et al. 2001). Finally, the produced spectra were binned to contain at least 50 and 30 photons per energy channel for the radioloud and radio-quiet sources, respectively, in order to have a sufficient signal to noise ratio and to allow the use of the $\chi^{2}$ statistics for the fit.

To perform the spectral analysis of RGS data we have used the standard science data files created by the RGS Pipeline Processing binned to contain at least 30 photons per energy channel.

\subsection{PKS 2149-306}

With the above selection criteria we obtained a total of $\gtrsim 43200$ net counts from the PN camera for a spectral fit. The hardness ratios of the counts in the $0.2-1 \mathrm{keV} / 2.5-10 \mathrm{keV}$ band show slight variations over the observation, not obviously correlated with the count rate. We therefore checked the spectral changes by dividing the observation interval in three parts. In all three intervals the fitted spectral power law slopes, assuming galactic $N_{\mathrm{H}}$ or fitting the absorbing column density, remained the same inside the statistical uncertainties. The differences were marginal and therefore we combined the whole data set for the spectral analysis.

We first fitted the PN data (see Table 2) with a simple power law model with free absorption for the $0.2-8 \mathrm{keV}$ energy range, leaving out the inherently noisy data above $8 \mathrm{keV}$. The fit and the resulting residuals, as ratio between model and data, are given in Fig. 2. This fit yielded a photon index $\Gamma=1.53 \pm 0.02$ and $N_{\mathrm{H}}=(2.94 \pm 0.32) \times 10^{20} \mathrm{~cm}^{-2}$, slightly in excess of the galactic value of $N_{\mathrm{H}}^{\mathrm{gal}}=2.10 \times 10^{20} \mathrm{~cm}^{-2}$. The fit is acceptable with a reduced $\chi_{\text {red }}^{2}=1.08 / 528$ d.o.f. A similar fit with $N_{\mathrm{H}}$ fixed to the galactic value is worse with $\Gamma=1.49 \pm 0.01$ and a reduced $\chi^{2}=1.11$. We further tried a broken power law model and obtained a very flat slope $\left(\Gamma_{\text {soft }}=1.03\right)$ at low energies, a slope of $\Gamma_{\text {hard }}=1.50$ at high energies, similar to the value of the single power law fit, a break energy of $E_{\text {break }}=0.7 \mathrm{keV}$, but with an $N_{\mathrm{H}}$ lower than the galactic value, with a reduced $\chi_{\text {red }}^{2}=1.04 / 526$. Fixing the absorption at the galactic value yields an equally acceptable fit $\left(\chi_{\text {red }}^{2}=1.05 / 527\right)$ with nearly identical parameters, except for a steeper slope $\left(\Gamma_{\text {soft }}=1.27\right)$ at low energies. An F-test gives an improvement for the broken power law fit at only the $\sim 86 \%$ confidence level, therefore we will from now on only consider single power law fits. 


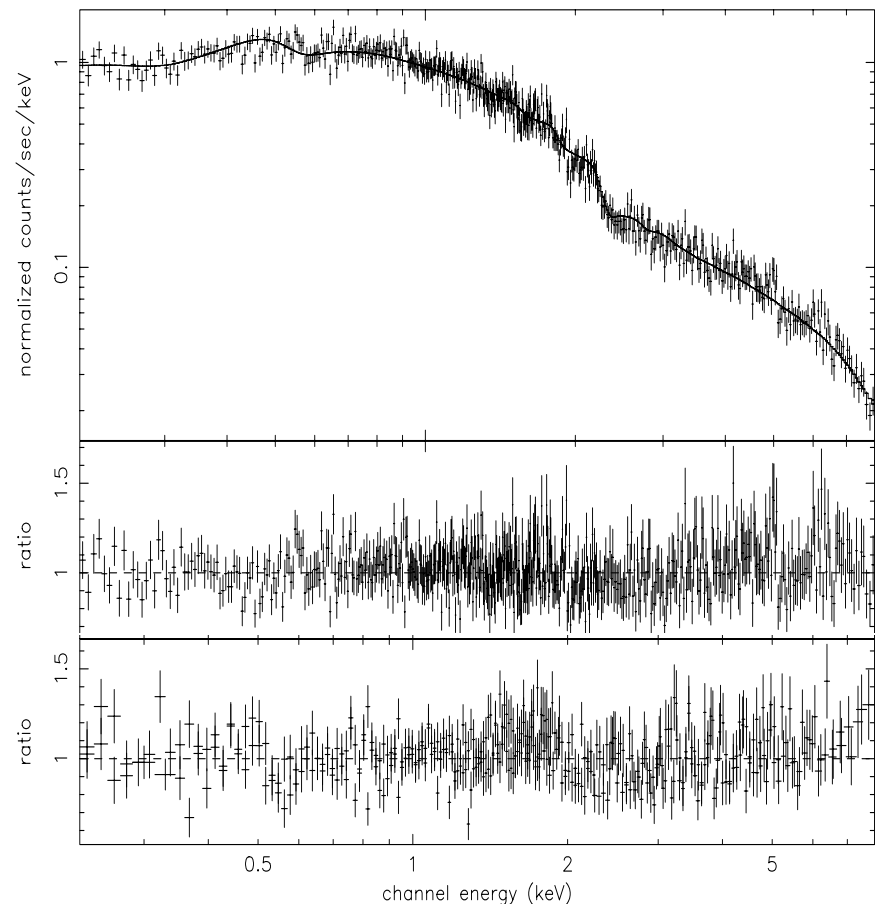

Fig. 2. Power law fit with free absorption for PKS 2149-306 in the $0.2-8 \mathrm{keV}$ energy range. The upper panel shows the PN fit, the middle panel the ratio between data and model; the lower panel gives for a comparison the ratios for the power law fit to the combined MOS1 plus MOS2 data.

The power law fit to the MOS data gave slightly different results, summarized in Table 2 . As can be seen, the reduced $\chi^{2}$ are larger for MOS2 and for both MOS1 and MOS2 the fitted slopes are considerably flatter than those for the PN, more than usually found when comparing MOS and PN data. Deviations from a simple power law spectrum could be responsible for the observed differences. For the MOS2 we also get a column density lower than the galactic value.

To improve the statistics, we combined the MOS1 and MOS2 data. The power law fits yielded similar results as above, with slopes still flatter than obtained for the PN (see Fig. 2). Interestingly, the slopes found for the simple power law fit to the MOS data are very similar to the values of the low-energy slopes of the broken power law fit to the PN data (with galactic $N_{\mathrm{H}}$ ).

However, the quality of the MOS data appears to be lower, the residuals are much noisier and the obtained $\chi^{2} \mathrm{~s}$ are worse than those from the fits to the PN data, which can be seen in Fig. 2 where the lower panel shows the ratios for the MOS fit. Whether remaining calibration uncertainties or an intrinsic source spectrum which cannot adequately be described by a simple power law, account for the observed discrepancies cannot be distinguished with the current data.

We further fitted the RGS data with an absorbed power law in the $0.35-2 \mathrm{keV}$ energy range. The fits to the RGS1 and RGS2 data yielded different results, with power law slopes $\Gamma=1.16 \pm 0.18\left(\chi_{\text {red }}^{2}=1.18 / 37\right.$ d.o.f. $)$ and $\Gamma=1.64 \pm 0.38$ $\left(\chi_{\text {red }}^{2}=0.91 / 43\right.$ d.o.f. $)$ assuming galactic absorption for RGS 1 and RGS2, respectively. The fits with free absorption gave
Table 2. Results for power law fits for PKS 2149-306 in the energy band $0.2-8.0 \mathrm{keV}$.

\begin{tabular}{lcccc}
\hline \hline Detector & $\begin{array}{c}N_{\mathrm{H}} \\
(1)\end{array}$ & $\Gamma$ & $\begin{array}{c}\text { Normalization } \\
(2)\end{array}$ & $\chi_{\text {red }}^{2} /$ d.o.f. \\
\hline PN & $2.94 \pm 0.32$ & $1.53 \pm 0.02$ & $0.87 \pm 0.15$ & $1.08 / 528$ \\
PN & galactic & $1.49 \pm 0.01$ & $0.83 \pm 0.07$ & $1.11 / 529$ \\
MOS1 & $2.34 \pm 0.80$ & $1.27 \pm 0.03$ & $0.95 \pm 0.03$ & $1.08 / 188$ \\
MOS1 & galactic & $1.27 \pm 0.02$ & $0.94 \pm 0.02$ & $1.07 / 189$ \\
MOS2 & $0.55 \pm 0.41$ & $1.27 \pm 0.03$ & $0.91 \pm 0.03$ & $1.28 / 189$ \\
MOS2 & galactic & $1.33 \pm 0.02$ & $0.97 \pm 0.02$ & $1.34 / 190$ \\
MOS1+MOS2 & $1.36 \pm 0.52$ & $1.27 \pm 0.03$ & (a) & $1.23 / 383$ \\
MOS1+MOS2 & galactic & $1.30 \pm 0.02$ & (a) & $1.24 / 384$ \\
\hline
\end{tabular}

The errors given are at the $90 \%$ level.

(1) In units of $10^{20} \mathrm{~cm}^{-2} ; N_{\mathrm{H}}^{\text {gal }}=2.10 \times 10^{20} \mathrm{~cm}^{-2}$.

(2) Normalization at $1 \mathrm{keV}$ in $10^{-3} \mathrm{ph} / \mathrm{keV} / \mathrm{cm}^{2} / \mathrm{s}$.

(a) Different normalizations for the individual detectors.

similar slopes with $N_{\mathrm{H}}$ consistent with the galactic value for the RGS1 whereas in the case of the RGS2 a flatter slope ( $\Gamma=1.52 \pm 0.50)$ with $N_{\mathrm{H}}$ lower than the galactic value is found. Due to the discrepancies between the two instruments, and to improve the photon statistics, we combined the RGS1 and RGS2 data, tolerating some data degradation and inferior resolution compared to the separate fits. The results yielded flat slopes, in between those from the analogous fits to the MOS and $\mathrm{PN}$ data $\left(\Gamma=1.41 \pm 0.13, \chi_{\text {red }}^{2}=1.14 / 81\right.$ d.o.f. for galactic $N_{\mathrm{H}} ; \Gamma=1.26 \pm 0.32, \chi_{\text {red }}^{2}=1.15 / 80$ d.o.f. with an $N_{\mathrm{H}}$ value compatible with zero). There are no obvious structures to be seen in the residuals, but the quality of the RGS data is rather low. Considering the discrepancies among the various instruments, we did not attempt to combine and fit the PN and MOS data or the PN and RGS data together, but rely in the following mainly on the PN data.

We tried other models like a thermal bremsstrahlung and a constant density ionized disk model (Ballantyne et al. 2001). The thermal bremsstrahlung model yielded a temperature of $k T=9.8 \mathrm{keV}$; however, the fit is unacceptable with a reduced $\chi_{\text {red }}^{2}=1.42 / 529$ d.o.f. The ionized disk model (assuming galactic $N_{\mathrm{H}}$ ) resulted in an ionization parameter $\xi=1.024$, a photon index of the incident power law $\Gamma=1.5$, a reflection fraction $R=1.73 \times 10^{-7}$, and a reduced $\chi_{\text {red }}^{2}=1.13 / 527$ d.o.f. Thus, the ionized disk model provides only a poor fit, the reflection fraction is very small, and the obtained slope is very similar to that of the power law fit.

Single power law fits to the data in the hard energy band $(\gtrsim 2 \mathrm{keV}$ ) result in excellent fits with slightly flatter slopes (see Table 3). An extrapolation of those power laws to lower energies seem to indicate a deficit of flux below $\sim 1 \mathrm{keV}$. However, small changes in the energy range used for the fit (especially when the inherently noisy data $\gtrsim 8 \mathrm{keV}$ are left out from the fit) result in acceptable fits $\left(\chi_{\text {red }}^{2} \sim 1\right)$ with slightly differing power law slopes and fitted $N_{\mathrm{H}}$ values in accordance to the galactic absorption.

The addition of an extra emission component to the power law, for example a black body with $k T=0.25 \pm 0.02 \mathrm{keV}$, 
Table 3. PN spectral fits for PKS 2149-306 in the hard energy band 2-8 keV and galactic absorption.

\begin{tabular}{lcccc}
\hline \hline \multicolumn{1}{c}{ Model } & $\Gamma$ & $E$ & $\sigma / \tau$ & $\chi_{\text {red }}^{2} /$ d.o.f. \\
& & $(1)$ & $(2)$ & \\
\hline pow & $1.44 \pm 0.04$ & & & $0.87 / 196$ \\
pow + Gauss & $1.44 \pm 0.05$ & $4.96 \pm 0.06$ & $(0.01)$ & $0.84 / 194$ \\
pow + edge & $1.40 \pm 0.07$ & $5.10 \pm 0.20$ & $0.10 \pm 0.10$ & $0.85 / 194$ \\
\hline
\end{tabular}

The errors given are at the $90 \%$ level.

(1) Line energy in $\mathrm{keV}$; the normalization of the line is $3.02 \pm 2.57 \times$ $10^{-6} \mathrm{ph} / \mathrm{keV} / \mathrm{cm}^{2} / \mathrm{s}$.

${ }^{(2)}$ Line width $(\sigma)$ or depth of the absorption edge $(\tau)$ in $\mathrm{keV}$.

contributing about 5-8\% to the flux at $1 \mathrm{keV}$, provides an acceptable fit over the whole $0.2-10.0 \mathrm{keV}$ energy band $\left(\chi_{\text {red }}^{2}=\right.$ $1.036 / 528$ d.o.f.) with a power law slope of $\Gamma=1.43 \pm 0.02$ and fixed galactic absorption.

Finally, we tried a power law with fixed galactic absorption over the whole energy band, allowing for extra absorption at the redshift of the source. The fit is acceptable $\left(\chi_{\text {red }}^{2}=\right.$ $1.065 / 528$ d.o.f.) with $\Gamma=1.53 \pm 0.02$ and an intrinsic column density of $N_{\mathrm{H}, z}=(1.09 \pm 0.04) \times 10^{21} \mathrm{~cm}^{-2}$. A similar fit could be achieved with galactic absorption plus an intrinsic warm absorber (absori model in XSPEC). The fit is acceptable $\left(\chi_{\text {red }}^{2}=1.067 / 527\right.$ d.o.f. $)$ with $\Gamma=1.53 \pm 0.03$. The column density of the warm absorber $N_{\text {warm }}=\left(1.31_{-0.9}^{+>3}\right) \times 10^{21} \mathrm{~cm}^{-2}$ is rather ill determined.

To investigate the presence of a Gaussian line around $\sim 5 \mathrm{keV}(\sim 17 \mathrm{keV}$ in the quasar rest frame) claimed by Yaqoob et al. (1999) from an ASCA observation, we restricted our analysis to the hard energy band (2-8 keV). We fitted a power law with galactic absorption and an additional narrow $(\sigma=0.01 \mathrm{keV})$ Gaussian line. The result for the fit is given in Table 3. The equivalent width of this narrow line would be about $12 \mathrm{eV}$ in the quasar's rest frame, much below the $\sim 300$ eV claimed by Yaqoob et al. (1999). Leaving the width of the line free in the fit resulted as well in a narrow line with nearly identical parameters and large errors. We further tried a power law fit with inclusion of an absorption edge at $\sim 5 \mathrm{keV}$ and obtained an equally acceptable fit (see Table 3).

Both, the fit with a Gaussian line and an absorption edge, improve the $\chi_{\text {red }}^{2}$ only marginally, the normalizations of the models are small and not well constrained and the improvements are statistically not significant. We also tried to add a Gaussian line to the model of a power law plus black body and galactic absorption, resulting in $k T=0.25 \mathrm{keV}, \Gamma=1.43$, a narrow line at $E=4.95 \mathrm{keV}$ and $\chi_{\text {red }}^{2}=1.03 / 525$ d.o.f. Also in this case the introduction of the line does not improve the fit significantly.

From the simple power law fit with free $N_{\mathrm{H}}$ in the whole energy band we obtain a $2-10 \mathrm{keV}$ flux and luminosity of $f_{2-10 \mathrm{keV}}=4.65 \times 10^{-12} \mathrm{erg} \mathrm{cm}^{-2} \mathrm{~s}^{-1}$ and $L_{2-10 \mathrm{keV}}=$ $1.03 \times 10^{47} \mathrm{erg} \mathrm{s}^{-1}$, respectively. For the ROSAT band we get $f_{0.1-2.4 \mathrm{keV}}=3.47 \times 10^{-12} \mathrm{erg} \mathrm{cm}^{-2} \mathrm{~s}^{-1}$ and $L_{0.1-2.4 \mathrm{keV}}=$ $7.73 \times 10^{46} \mathrm{erg} \mathrm{s}^{-1}$.

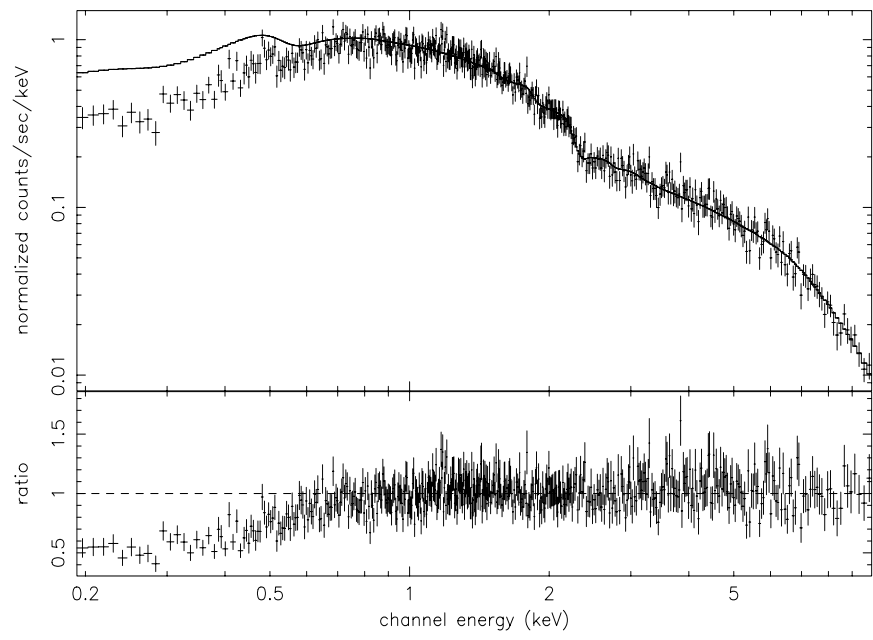

Fig. 3. Power law fit with galactic absorption for PKS 2126-158 over the restricted energy band $2-10 \mathrm{keV}$, extrapolated to lower energies.

\subsection{PKS $2126-158$}

Applying the same selection criteria as for PKS 2149-306, we collected a total of $\gtrsim 44300$ net counts from the PN camera. We started fitting a simple power law model in the $0.2-10.0 \mathrm{keV}$ range and obtained a photon index $\Gamma=1.51 \pm 0.02$, an $N_{\mathrm{H}}=1.1 \times 10^{21} \mathrm{~cm}^{-2}$, in excess of the galactic value and a $\chi_{\text {red }}^{2}=0.98 / 492$ d.o.f. (see Table 4). A single power law fit with fixed galactic absorption is not acceptable with $\chi_{\text {red }}^{2}=$ $1.85 / 493$ d.o.f.

A power law fit, limited to the hard energy band $(2.0-10.0 \mathrm{keV})$, with absorption fixed to the galactic value is acceptable $\left(\chi_{\text {red }}^{2}=0.88 / 197\right.$ d.o.f. $)$ with $\Gamma=1.43 \pm 0.04$. The extrapolation of this power law fit to lower energies (see Fig. 3) clearly demonstrates the necessity for more absorption at low energies.

The fit further indicates that, although excellent power law fits can be achieved, there might be spectral deviations from this simple model. As an indication for this we notice, that the same fit, limited to the hard energy band (3.0-10.0 keV), with fixed galactic absorption is acceptable $\left(\chi_{\text {red }}^{2}=0.99 / 133\right.$ d.o.f. $)$ with a different slope of $\Gamma=1.50 \pm 0.07$.

Fitting a power law over the whole energy band, fixing the galactic absorption and allowing for extra absorption at the redshift of the source, gives a good fit $\left(\chi_{\text {red }}^{2}=0.90 / 492\right.$ d.o.f. $)$ with extra $N_{\mathrm{H}, \mathrm{z}}=1.40 \times 10^{22} \mathrm{~cm}^{-2}$ and $\Gamma=1.47 \pm 0.02$. Allowing the redshift of the absorber to vary results in a value in agreement with the redshift of the quasar. We checked if a warm absorber could be responsible for the extra absorption, performing a fit with the absori model. The ratios between the data and the model of the resulting fit are shown in the lower panel of Fig. 4 with extra $N_{\mathrm{H}, \mathrm{z}}=1.35 \times 10^{22} \mathrm{~cm}^{-2}$ at the redshift of the source, $\Gamma=1.47 \pm 0.02$, the absorber temperature fixed at $T=3 \times 10^{4} \mathrm{~K}$ and $\chi_{\text {red }}^{2}=0.90 / 490$ d.o.f. However, the ionization parameter is not well constrained and the fit is statistically equivalent to the one with a neutral absorber at the source's redshift.

The results of the power law fits for the MOS data are shown in Table 4. As in the case of PKS 2149-306 the fits 
Table 4. Results for power law fits for PKS 2126-158.

\begin{tabular}{lcccc}
\hline \hline Detector & $\begin{array}{c}N_{\mathrm{H}} \\
(1)\end{array}$ & $\Gamma$ & $\begin{array}{c}\text { Normalization } \\
(2)\end{array}$ & $\chi_{\text {red }}^{2} /$ d.o.f. \\
& \multicolumn{1}{c}{ (1) } & & \\
\hline $\mathrm{PN}^{(a)}$ & 1.1206 & $1.51 \pm 0.02$ & $0.99 \pm 0.02$ & $0.98 / 492$ \\
$\mathrm{PN}^{(a)}$ & galactic & $1.30 \pm 0.01$ & $0.77 \pm 0.01$ & $1.85 / 493$ \\
$\mathrm{PN}^{(b)}$ & $0.49 \pm 8.28$ & $1.50 \pm 0.20$ & $1.00 \pm 0.49$ & $1.00 / 132$ \\
$\mathrm{PN}^{(b)}$ & galactic & $1.50 \pm 0.07$ & $1.00 \pm 0.12$ & $0.99 / 133$ \\
$\mathrm{PN}^{(c)}$ & $3.10 \pm 1.99$ & $1.49 \pm 0.41$ & $0.87 \pm 0.33$ & $0.87 / 196$ \\
$\mathrm{PN}^{(c)}$ & galactic & $1.43 \pm 0.04$ & $0.88 \pm 0.04$ & $0.88 / 197$ \\
$\mathrm{MOS1}^{(a)}$ & $1.29 \pm 0.09$ & $1.35 \pm 0.03$ & $1.74 \pm 0.06$ & $1.26 / 229$ \\
$\mathrm{MOS1}^{(a)}$ & galactic & $1.11 \pm 0.01$ & $1.31 \pm 0.02$ & $2.36 / 230$ \\
$\operatorname{MOS}^{(a)}$ & $1.13 \pm 0.09$ & $1.34 \pm 0.03$ & $1.71 \pm 0.06$ & $1.22 / 233$ \\
$\operatorname{MOS2}^{(a)}$ & galactic & $1.14 \pm 0.02$ & $1.36 \pm 0.02$ & $1.90 / 234$ \\
\hline
\end{tabular}

The errors given are at the $90 \%$ level.

(1) In units of $10^{21} \mathrm{~cm}^{-2} ; N_{\mathrm{H}}^{\mathrm{gal}}=4.90 \times 10^{20} \mathrm{~cm}^{-2}$.

(2) Normalization at $1 \mathrm{keV}$ in $10^{-3} \mathrm{ph} / \mathrm{keV} / \mathrm{cm}^{2} / \mathrm{s}$.

${ }^{(a)}$ Energy band $0.2-10.0 \mathrm{keV}$.

(b) Energy band $3.0-10.0 \mathrm{keV}$.

(c) Energy band 2.0-10.0 keV.

are systematically worse than those for the PN, with significantly flatter slopes and larger $\chi^{2}$ s. Apart from these differences, the MOS data show evidence for excess absorption in PKS 2126-158 as well. A simple power law fit with excess absorption doesn't provide a good description of the spectrum and the remaining residuals indicate a more complicated structure, but the photon statistics of the MOS data are insufficient for a more detailed modeling.

Fitting the same models to the RGS data gives results consistent with those obtained for the PN, but with flatter slopes and lower excess absorption $\left(\Gamma \sim 1.2, N_{\mathrm{H}}=8.7 \times 10^{20} \mathrm{~cm}^{-2}\right.$ for RGS1, $\Gamma \sim 1.4, N_{\mathrm{H}}=8.3 \times 10^{20} \mathrm{~cm}^{-2}$ for RGS2, $\Gamma \sim$ 1.2, $N_{\mathrm{H}}=6.6 \times 10^{20} \mathrm{~cm}^{-2}$ for the combined fit). The residuals show an excess of emission around $1.7 \mathrm{keV}$ which we further tried to model with a Gaussian line with fixed energy $(E=1.72 \mathrm{keV})$ and width $(\sigma=0.05 \mathrm{keV})$, with equivalent widths of $\sim 129 \mathrm{eV}$ for the RGS1 and $\sim 67 \mathrm{eV}$ for the RGS2. This allowed us to recover values of the parameters similar to the PN case both for the absorption $\left(N_{\mathrm{H}}=1.0 \times 10^{21} \mathrm{~cm}^{-2}\right)$ and the power law slope $(\Gamma=1.55 \pm 0.41)$. However, the rest frame energy of the line would be $\sim 7.3 \mathrm{keV}$, not immediately recognizable as any known feature.

We tried additional models relying again on the PN data alone. An ionized disk model yielded acceptable results with the following best fit parameters: $N_{\mathrm{H}}=1.2 \times 10^{21} \mathrm{~cm}^{-2}$, ionization parameter $\xi=1.044, \Gamma=1.56$, reflection fraction $R=0.11$, redshift $z=3.262$ (consistent with the source's redshift) and $\chi_{\text {red }}^{2}=0.97 / 489$ d.o.f. But the errors on the parameters are large so that this fit is not reliable.

A thermal bremsstrahlung model with free $N_{\mathrm{H}}$ gives a slightly worse fit than the power law $\left(\chi_{\text {red }}^{2}=1.11 / 491\right.$ d.o.f.), whereas a broken power law fit results in very low values of the soft photon index $\left(\Gamma_{\text {soft }}=0.09\right.$ for free $\left.N_{\mathrm{H}}\right)$ and huge errors.

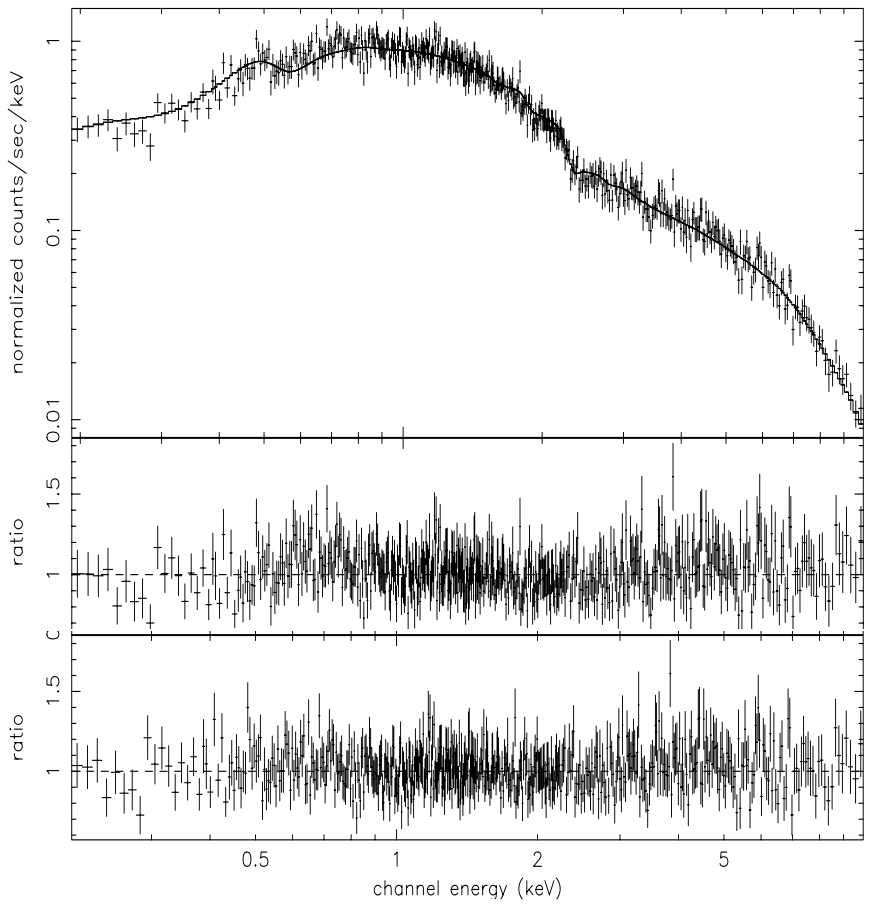

Fig. 4. Power law fit with free absorption to the PN data for PKS 2126-158 (upper and middle panel). The lower panel shows the ratios between data and model for the warm absorber fit with galactic absorption to the PN data.

Using the results from the simple power law fit with free absorption in the whole energy band we obtain the following fluxes and luminosities: $f_{2-10 \mathrm{keV}}=5.46 \times 10^{-12} \mathrm{erg} \mathrm{cm}^{-2} \mathrm{~s}^{-1}$, $L_{2-10 \mathrm{keV}}=2.23 \times 10^{47} \mathrm{erg} \mathrm{s}^{-1}, f_{0.1-2.4 \mathrm{keV}}=3.92 \times$ $10^{-12} \mathrm{erg} \mathrm{cm}^{-2} \mathrm{~s}^{-1}$ and $L_{0.1-2.4 \mathrm{keV}}=1.61 \times 10^{47} \mathrm{erg} \mathrm{s}^{-1}$.

\section{3. $Q 0000-263$}

From the $\gtrsim 40 \mathrm{ksec}$ observation we collected only $\gtrsim 1400$ net source counts from the PN camera. We tried simple power law fits in the whole energy band for the PN and MOS cameras separately. In all three cases the fitted $N_{\mathrm{H}}$ was consistent with the galactic value, so we fixed it to reduce the number of fit parameters. The resulting photon indices are given in Table 5. As they are consistent with each other within the errors, we combined the PN and MOS spectra to improve the statistics. A simple power law fit with galactic absorption yielded a photon index $\Gamma=2.10 \pm 0.11$ and $\chi_{\text {red }}^{2}=0.85 / 110$ d.o.f. (see Fig. 5). No Fe line (expected at $\sim 1.2 \mathrm{keV}$ in the observer's frame) is required by the data. Thus, a simple power law with galactic absorption seems to be a sufficient model to describe the data. Some deviations are visible in the residuals at $\sim 0.9 \mathrm{keV}$, which we tried to model with a broad Gaussian line, however the line's parameters are not well constrained. The photon counts are insufficient to model this feature.

With the above parameters for the PN fits we obtain a $2-10 \mathrm{keV}$ flux of $f_{2-10 \mathrm{keV}}=2.55 \times 10^{-14} \mathrm{erg} \mathrm{cm}^{-2} \mathrm{~s}^{-1}$ which results in a luminosity of $L_{2-10 \mathrm{keV}}=4.82 \times 10^{45} \mathrm{erg} \mathrm{s}^{-1}$ of the source. In the ROSAT band we get $f_{0.1-2.4 \mathrm{keV}}=7.76 \times$ $10^{-14} \mathrm{erg} \mathrm{cm}^{-2} \mathrm{~s}^{-1}$ and $L_{0.1-2.4 \mathrm{keV}}=1.46 \times 10^{46} \mathrm{erg} \mathrm{s}^{-1}$, 
Table 5. Results for power law fits and galactic absorption $\left(N_{\mathrm{H}}^{\mathrm{gal}}=\right.$ $1.67 \times 10^{20} \mathrm{~cm}^{-2}$ ) for Q 0000-263 in the energy band $0.2-9.0 \mathrm{keV}$.

\begin{tabular}{lccc}
\hline \hline Detector & $\Gamma$ & $\begin{array}{c}\text { Normalization } \\
(1)\end{array}$ & $\chi_{\text {red }}^{2}$ /d.o.f. \\
\hline PN & $2.19 \pm 0.12$ & $1.31 \pm 0.14$ & $0.91 / 64$ \\
MOS1 & $1.98 \pm 0.40$ & $1.58 \pm 0.19$ & $0.67 / 21$ \\
MOS2 & $1.82 \pm 0.23$ & $1.46 \pm 0.23$ & $0.52 / 23$ \\
PN+MOS & $2.10 \pm 0.11$ & (a) & $0.85 / 110$ \\
\hline
\end{tabular}

The errors given are at the $90 \%$ level.

(1) Normalization at $1 \mathrm{keV}$ in $10^{-5} \mathrm{ph} / \mathrm{keV} / \mathrm{cm}^{2} / \mathrm{s}$.

(a) Different normalizations for the individual detectors.

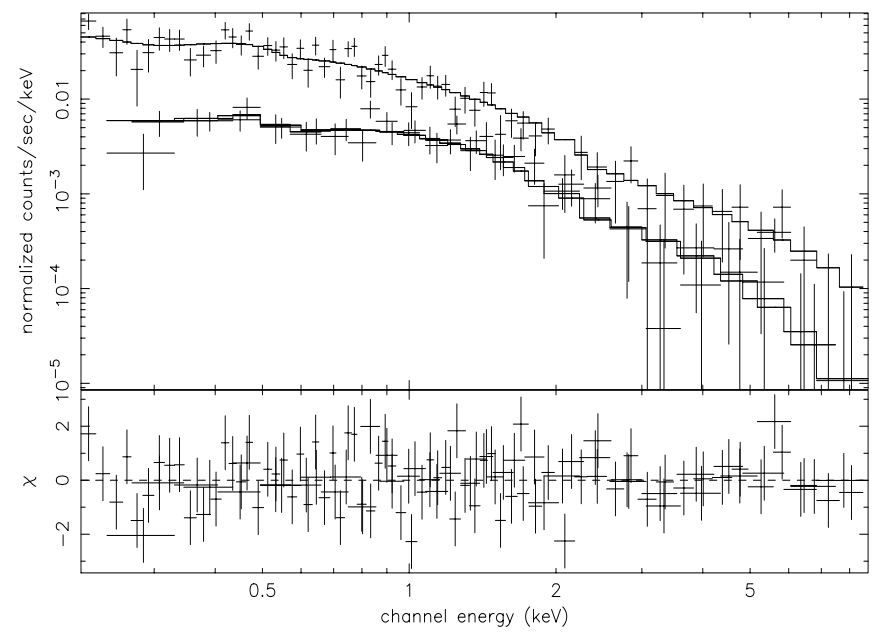

Fig. 5. Combined PN+MOS power law fit with galactic absorption for Q $0000-263$ in the $0.2-9 \mathrm{keV}$ energy range. The lower panel gives the $\Delta \chi^{2}$ per channel.

consistent with the values found by Bechtold et al. (1994a) and Kaspi et al. (2000) within the errors.

\section{4. $Q 1442+2931$}

A total of $\gtrsim 3800$ net counts were collected for the source in the PN camera. Again, we first tried simple power law fits for the PN and MOS instruments separately, which resulted in absorption column densities consistent with the galactic value. We therefore fixed the $N_{\mathrm{H}}$ to the galactic value and obtained the results given in Table 6.

The fit to the very noisy MOS 1 data gave a high $\chi_{\text {red }}^{2}$, in contrast to the fit for the MOS2 camera. The MOS1 data could also be responsible for the high $\chi_{\text {red }}^{2}$ of the combined PN + MOS fit (see Fig. 6). All fits indicate that a power law describes the spectrum of the source quite well and that no excess absorption is required. The MOS data, but not the PN data, seem to require an extra emission component at $\sim 1.5 \mathrm{keV}$, which we tried to model as a broad Gaussian line. From the fit of the co-added MOS1 and MOS2 data we obtained a line energy $E=1.48 \pm 0.12 \mathrm{keV}$; however the line width is not constrained and the fit doesn't improve significantly after the addition of the
Table 6. Results for power law fits and galactic absorption $\left(N_{\mathrm{H}}^{\mathrm{gal}}=\right.$ $1.56 \times 10^{20} \mathrm{~cm}^{-2}$ ) for Q $1442+2931$ in the energy band $0.2-9.0 \mathrm{keV}$.

\begin{tabular}{lccc}
\hline \hline Detector & $\Gamma$ & $\begin{array}{c}\text { Normalization } \\
(1)\end{array}$ & $\chi_{\text {red }}^{2} /$ d.o.f. \\
\hline PN & $1.92 \pm 0.07$ & $4.31 \pm 0.30$ & $0.87 / 105$ \\
MOS1 & $1.82 \pm 0.11$ & $5.19 \pm 0.48$ & $2.03 / 48$ \\
MOS2 & $1.76 \pm 0.13$ & $5.15 \pm 0.51$ & $0.84 / 48$ \\
PN+MOS & $1.87 \pm 0.05$ & (a) & $1.14 / 203$ \\
\hline
\end{tabular}

The errors given are at the $90 \%$ level.

(1) Normalization at $1 \mathrm{keV}$ in $10^{-5} \mathrm{ph} / \mathrm{keV} / \mathrm{cm}^{2} / \mathrm{s}$.

(a) Different normalizations for the individual detectors.

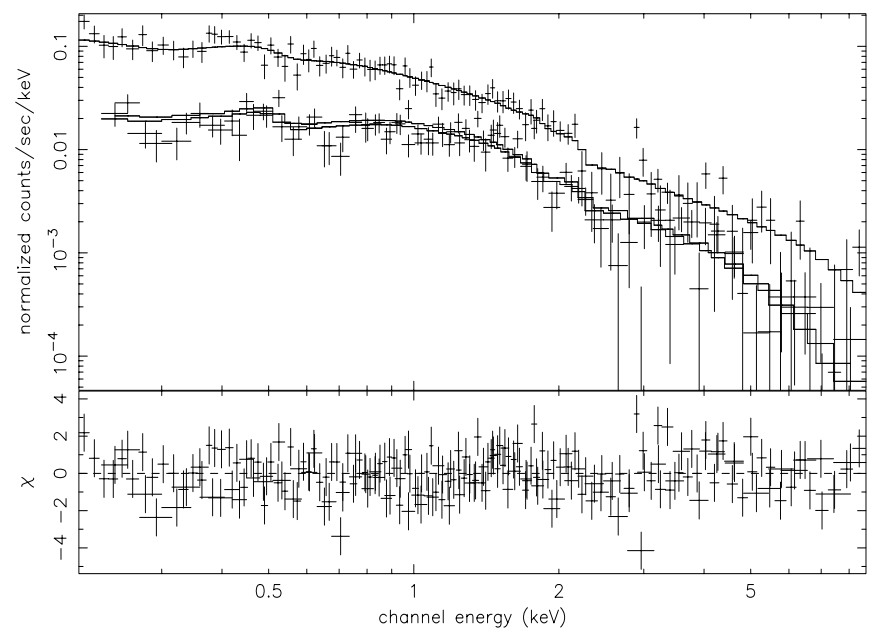

Fig. 6. Combined $\mathrm{PN}+\mathrm{MOS}$ power law fit with galactic absorption for Q $1442+2931$ in the $0.2-9 \mathrm{keV}$ energy range. The lower panel gives the $\Delta \chi^{2}$ per channel.

line. The low quality of the MOS data and the small number of counts argue against the real presence of this feature. The addition of the PN data leads to a shift of the free fitted line center to an energy below $0.2 \mathrm{keV}$, with large errors on both the energy of the line and its width. We note that a redshifted Fe line would be expected at $\sim 1.7 \mathrm{keV}$, where no feature is observed.

The $2-10 \mathrm{keV}$ flux and luminosity resulting from the PN fit are $f_{2-10 \mathrm{keV}}=1.25 \times 10^{-13} \mathrm{erg} \mathrm{cm}^{-2} \mathrm{~s}^{-1}$ and $L_{2-10 \mathrm{keV}}=$ $5.82 \times 10^{45} \mathrm{erg} \mathrm{s}^{-1}$, respectively. For the ROSAT band they are $f_{0.1-2.4 \mathrm{keV}}=2.08 \times 10^{-13} \mathrm{erg} \mathrm{cm}^{-2} \mathrm{~s}^{-1}$ and $L_{0.1-2.4 \mathrm{keV}}=$ $9.64 \times 10^{45} \mathrm{erg} \mathrm{s}^{-1}$, consistent inside the errors with no flux variations between the ROSAT (Reimers et al. 1995) and XMM observations ten years apart.

\section{Discussion}

We have presented the results of detailed spectral analysis of two high $-z$, very luminous, radio-loud quasars and two high $-z$ radio-quiet quasars. XMM's high sensitivity and wide energy band allowed a reliable determination of the quasars' spectra over a source intrinsic energy band up to $\sim 50 \mathrm{keV}$ and to address the question of intrinsic absorption in the objects. 


\subsection{Radio-loud objects}

A simple power law with a slope of $\Gamma \sim 1.53$ provides a reasonably good fit to the data from PKS 2149-306, with absorption near the galactic value. However, the fact that MOS and PN data yield different slopes, that fits over restricted energy ranges for the same instrument yield slightly different power law indices, and that the residuals show some "systematic" variations indicates, that a simple power law is perhaps not the best description for the spectrum. But the deviations from a straight power law are small and statistically only separable by increasing the signal to noise ratio in a longer observation.

Depending on the models fitted to the data the results for the absorption column density change slightly. Adding a small black body component with $k T \sim 0.25 \mathrm{keV}$, which might be a reflection component in the rest frame of the source, to the power law gives an acceptable fit with galactic absorption. Another interesting possibility could be that we are seeing the soft X-ray bump, never observed up to now, produced in blazars by the Comptonization of external UV radiation by electrons in the jet (Sikora et al. 1997).

Source intrinsic, extra warm or cold absorption models predict a column density of $\sim 10^{21} \mathrm{~cm}^{-2}$ in the rest frame of the source. Considering the quality of the available data we conclude, however, that there is no strong evidence for substantial absorption in excess of the galactic value towards PKS 2149-306.

The source was previously observed by ROSAT and ASCA (Siebert et al. 1996; Cappi et al. 1997), BeppoSAX (Elvis et al. 2000), and Chandra (Fang et al. 2001). A comparison between the different instruments indicates spectral and flux variability. The ASCA power law slopes are comparable to ours and the spectra show indications of excess absorption of a few times $10^{20} \mathrm{~cm}^{-2}$. BeppoSAX claims a broken power law with a similar flat slope and soft excess emission below $\sim 0.8 \mathrm{keV}$, while the Chandra spectra are in agreement with galactic absorption, and a very flat power law slope $(\Gamma \sim 1.26)$. The $2-10 \mathrm{keV}$ flux measured in October 1994 by ASCA is about 30\% higher than that obtained by Chandra, BeppoSAX (in 1997), and XMM $\left(\sim 7-8 \times 10^{-12} \mathrm{erg} \mathrm{cm}^{-2} \mathrm{~s}^{-1}\right)$. The RASS flux in 1990/91 seems to have been a factor of two lower (Schartel et al. 1996). However, the rather uncertain spectral slope makes an exact comparison problematic.

In none of the recent observations (BeppoSAX, Chandra, and XMM-Newton) the claimed detection of a line at $\sim 5 \mathrm{keV}$ could be confirmed, nor is there evidence for line emission at other energies.

PKS 2126-158 clearly shows the presence of extra absorption of the order of $\Delta N_{\mathrm{H}} \gtrsim 6 \times 10^{20} \mathrm{~cm}^{-2}$. At the redshift of the source this corresponds to $N_{\mathrm{H}, z} \sim 1.4 \times 10^{22} \mathrm{~cm}^{-2}$ and a power law model with galactic absorption plus an intrinsic cold or a warm absorber, results in excellent fits.

The object has been observed four times between 19911993 with the ROSAT PSPC (Elvis et al. 1994b; Cappi et al. 1997) and in 1993 by ASCA (Cappi et al. 1997). For all observations the fitted power law slopes and the values of excess absorption are consistent with our results inside the errors with no indications of temporal variations. The measured fluxes, even from the early Einstein IPC observation (Worrall $\&$ Wilkes 1990) are identical inside the statistical uncertainties, $f_{2-10 \mathrm{keV}} \sim 1 \times 10^{-11} \mathrm{erg} \mathrm{cm}^{-2} \mathrm{~s}^{-1}$. The constancy of this flux level is relatively unusual for such a bright radio-loud quasar.

\subsubsection{X-ray and broad band spectral properties}

The power law fits with free absorption in the whole energy band yielded for both quasars a slope $\Gamma \lesssim 1.5$. This value is typical of those from Einstein observations (Wilkes \& Elvis 1987) but is at the lower limit of the range commonly found for radio-loud quasars from ROSAT and ASCA observations (Brinkmann et al. 1997; Reeves \& Turner 2000). Similar flat slopes for PKS 2149-306 and PKS 2126-158 were reported from previous ASCA and ROSAT observations (Elvis et al. 1994b; Cappi et al. 1997; Siebert et al. 1996) and in a Chandra observation Fang et al. (2001) found an even flatter slope for PKS 2149-306.

In the frame of the two-emission components model an explanation would be that the emission is dominated by the beamed, flat blazar-like component. The high X-ray luminosities of the two objects $\left(L_{2-10 \mathrm{keV}} \simeq 2 \times 10^{47} \mathrm{erg} \mathrm{s}^{-1}\right.$ and $L_{2-10 \mathrm{keV}} \simeq 7 \times 10^{46} \mathrm{erg} \mathrm{s}^{-1}$ for PKS 2126-158 and PKS 2149-306, respectively) would be in accordance with this hypothesis. In the case of PKS 2126-158 we also find indication of beaming in the extremely high radiative efficiency ( $\eta \gtrsim 8$ ) of accretion onto a black hole exceeding the maximum theoretical value.

In order to get more insight into the properties of our sources we calculated the broad band spectral indices between $5 \mathrm{GHz}, 2500 \AA$ and $2 \mathrm{keV}$, which are good indicators of the SED's shape. The values we obtained are $\alpha_{\mathrm{ox}}=1.11$, $\alpha_{\mathrm{rx}}=0.81, \alpha_{\mathrm{ro}}=0.67$ for PKS 2126-158, and $\alpha_{\mathrm{ox}}=0.95$, $\alpha_{\mathrm{rx}}=0.81, \alpha_{\mathrm{ro}}=0.74$ for PKS 2149-306. Considering the uncertainty on the X-ray fluxes $(\$ 30 \%)$ resulting from the errors of the spectral fit parameters we estimate a typical error for the $\alpha_{\text {ox }}$ and $\alpha_{\text {rx }}$ of the order of $\$ 5 \%$. Padovani et al. (2002) recently studied a sample of FSRQ characterized by a synchrotron peak at X-ray energies in the same way as HBL BL Lacs. We checked if our sources could belong to this class and thus explain their X-ray loudness. However, the above values of the broad band spectral indices place our sources in the "normal" FSRQ region (see Fig. 1 of Padovani et al. 2002), pointing at an inverse Compton origin for the X-ray emission from the sources. Indeed, PKS 2149-306 is known to have a blazar-like SED (Elvis et al. 2000) with the synchrotron peak at $\sim 0.3 \mathrm{~mm}$ and the inverse Compton peak at $\sim 4 \mathrm{MeV}$, providing strong support to its FSRQ classification.

On the other hand, PKS $2126-158$ is known to be a GPS source (de Vries et al. 1997; Stanghellini et al. 1998). These powerful radio sources are characterized by extreme compactness $(\$ 1 \mathrm{kpc})$, low polarization and a convex spectrum at radio frequencies with a turn-over between $\sim 500 \mathrm{MHz}$ and $\sim 10 \mathrm{GHz}$. This characteristic shape of the spectrum is commonly interpreted in terms of synchrotron self-absorption (O' Dea 1998). Due to their double-lobed radio morphologies, GPS sources are thought to be lying in the plane of the sky, so that beaming 
shouldn't play any role. This makes the tentative classification of PKS 2126-158 as a FSRQ rather problematic as already discussed by Elvis et al. (1994b). However, new VLBA observations of several GPS sources (Lister et al. 2002) revealed some atypical features for this class of objects, like core-jet structures, super-luminal motion, variability and polarization and this, together with the radiative efficiency argument (see above), suggest relativistic beaming at least for some of the GPS sources. Moreover, these properties seem to agree better with a free-free absorption model from a surrounding ionized medium than with the synchrotron absorption model (Lister et al. 2002). Interestingly, this absorbing medium could also account for the extra absorption we detect in PKS 2126-158 (see below).

\subsubsection{Absorption properties}

Although excess absorption was previously reported (Cappi et al. 1997; Siebert et al. 1996) for PKS 2149-306, we find no strong evidence for extra absorption for this source. Depending on the assumed spectral form the fits predict absorbing column densities ranging from the galactic value to an additional source intrinsic value of $\Delta N_{\mathrm{H}, z} \simeq 1.1 \times 10^{21} \mathrm{~cm}^{-2}$ in case of a single power law fit over the whole energy range. As the residuals of the fits indicate a more complex spectral form than a simple power law the existence of any strong extra absorption must be regarded as uncertain.

For PKS 2126-158 we find additional absorption of $\Delta N_{\mathrm{H}} \simeq$ $0.6 \times 10^{21} \mathrm{~cm}^{-2}$ in excess of the galactic value. For this object extra absorption has been claimed before from ASCA and ROSAT observations (Elvis et al. 1994; Serlemitsos et al. 1994; Cappi et al. 1997; Reeves \& Turner 2000). Their results are in agreement with ours.

The absence of extra absorption in PKS 2149-306 argues against its ubiquity in high redshift quasars (Yuan \& Brinkmann 1998). On the other hand, the presence of extra absorption in PKS 2126-158 rises the question of the location of the absorbing material. In fact, our fits are compatible with an absorber at the redshift of the source, but other redshifts are equally allowed by the data, so that this issue remains open.

A galactic origin of the extra absorption by means of molecular clouds and dust has been excluded for this source by local CO surveys and IRAS measurements (Cappi et al. 1997). The absorption could then be due to intervening matter along the line of sight. Damped Ly $\alpha$ systems with column densities of the order of $10^{20}-10^{21} \mathrm{~cm}^{-2}$ or intervening galaxies could provide the necessary amount of absorption.

However, no damped Ly $\alpha$ systems have been detected in the optical spectrum of PKS 2126-158, but only the Ly $\alpha$ forest (Giallongo et al. 1993) with much lower column densities $\left(N_{\mathrm{H}} \sim 10^{16}-10^{17} \mathrm{~cm}^{-2}\right)$, which cannot account for the observed absorption. Moreover, some absorption variability seems to be present in PKS 2126-158, not explicable by damped Ly $\alpha$ systems, which are believed to be stable over long time scales.

It thus seems likely that the absorber is at the quasar's position. Then a possible site for the absorber could be the torus invoked in the unified model for AGNs or, allowing for variable absorption, dusty clouds. A further alternative could be a cooling flow in a cluster of galaxies. As PKS 2126-158 is classified as a GPS source, an interesting possibility is that the proposed ionized medium (Lister et al. 2002) responsible for the GPS phenomenon through free-free absorption, could be the same absorbing medium we detect in the X-ray band.

Our data do not allow to distinguish between a cold, neutral and a warm, ionized absorber as we cannot see in the spectrum the typical features of a warm absorber, i.e. the OVII and OVIII edges at $\sim 0.7-1.0 \mathrm{keV}$ and an extra emission component below $\sim 0.7 \mathrm{keV}$ in the rest frame; these energies are redshifted below $\sim 0.2 \mathrm{keV}$, outside the XMM band. However, if the absorbing matter can be related to the $\operatorname{Ly} \alpha$ clouds along the line of sight the temperature of the gas as determined from high resolution optical observations (D'Odorico et al. 1998) is around $T \sim 38000 \mathrm{~K}$. The findings of Brinkmann et al. (1997) that the majority of the high redshift objects in their sample are GPS sources and that they show excess absorption further supports the connection between the X-ray absorber and the conceivable free-free absorber of Lister et al. (2002).

\subsection{Radio-quiet objects}

A simple power law fit to the combined PN + MOS data of Q 0000-263 gives a statistically acceptable representation of the spectrum and no absorption in excess of the galactic is found. Unfortunately, the available number of photons is insufficient for a more complex modeling of the remaining residuals. Our findings are consistent with those from previous ROSAT observations within the errors. We obtain from the combined $\mathrm{PN}+\mathrm{MOS}$ fit a photon index of $\Gamma=2.10 \pm 0.11$, whereas Bechtold et al. (1994) find for the 0.1-2.4 keV band a spectral index $\alpha=1.30 \pm 0.23$ and galactic absorption. From this fit they deduced a broad spectral index $\alpha_{\mathrm{ox}}=1.85$ whereas we find a slightly different value $\alpha_{\mathrm{ox}}=1.78$. Kaspi et al. (2000) give a broad spectral index $\alpha_{\text {ox }}=1.65$. The errors on the $\alpha_{\text {ox }}$ have been estimated to be $\$ 5 \%$ as in Sect. 4.1.1. The discrepancies in these values can be explained by the different spectral slopes used in the calculations and the restricted ROSAT energy range compared to XMM.

Similar results hold for Q $1442+2931$, for which an acceptable fit is again provided by a simple power law and galactic absorption. The power law slope for the combined PN+MOS fit is $\Gamma=1.87 \pm 0.05$. Due to the low number of counts no spectral analysis of ROSAT observations could be performed by Reimers et al. (1995) for a comparison with our results but the deduced fluxes are consistent inside their errors. For this quasar we obtain a broad spectral index $\alpha_{\mathrm{ox}}=1.70$.

The power law slopes we get for the two high redshift radioquiet quasars are in agreement with the typical values found at low redshifts (Yuan et al. 1998a), suggesting the absence of spectral evolution for this class of AGN and the presence of a constant spectral form over a wide band width. Further, the slopes are also considerably steeper than those of the two radioloud quasars of our sample, supporting the spectral dependence 
Table 7. Summary of source properties.

\begin{tabular}{lcccccccc}
\hline \hline \multicolumn{1}{c}{ Source } & type & $z$ & $m_{V}$ & $\begin{array}{c}\alpha_{\mathrm{ox}} \\
(1)\end{array}$ & $\begin{array}{c}\Gamma \\
(2)\end{array}$ & $\begin{array}{c}L_{2-10 \mathrm{keV}} \\
{\left[\times 10^{47} \mathrm{erg} / \mathrm{s}\right]}\end{array}$ & $\begin{array}{c}N_{\mathrm{H}} \\
(3)\end{array}$ & $\chi_{\text {red }}^{2} /$ d.o.f. \\
\hline PKS 2149-306 & RL & 2.34 & 18.5 & 0.95 & $1.53 \pm 0.02$ & 1.03 & 0.294 & $1.08 / 528$ \\
PKS 2126-158 & RL & 3.27 & 17.0 & 1.11 & $1.51 \pm 0.02$ & 1.97 & 1.1 & $0.98 / 492$ \\
Q 1442+2931 & RQ & 2.64 & 17.0 & 1.70 & $1.92 \pm 0.07$ & 0.06 & galactic & $0.87 / 105$ \\
Q 0000-263 & RQ & 4.10 & 18.0 & 1.78 & $2.19 \pm 0.12$ & 0.05 & galactic & $0.91 / 64$ \\
\hline
\end{tabular}

The errors given are at the $90 \%$ level.

(1) Estimated error of $\$ 5 \%$.

(2) Power law slope of fit to PN data with free absorption.

(3) Fitted absorption in units of $10^{21} \mathrm{~cm}^{-2}$.

on the radio-loud/radio-quiet classification observed at low redshifts (Brinkmann et al. 1997; Yuan et al. 1998a).

The $\alpha_{\text {ox }}$ for these two high redshift quasars are consistent with values found in previous studies for $z>2$ and they are larger than the average values found at $z<0.2$ (Yuan et al. 1998a). This indicates that high redshift radio-quiet quasars are more X-ray quiet than their low-redshift counterparts. However, it is found (Avni \& Tananbaum 1982; Avni \& Tananbaum 1986; Wilkes et al. 1994; Yuan et al. 1998a) that the X-ray loudness is independent of redshift and that instead a correlation is present between $\alpha_{\mathrm{ox}}$ and $\log l_{\mathrm{o}}$, where $l_{\mathrm{o}}$ is the luminosity at $2500 \AA$. Thus the larger $\alpha_{\text {ox }}$ would arise from a higher $l_{\mathrm{o}}$ at high redshift for these optically selected quasars. From the broad spectral indices it is also inferred that radioloud quasars are X-ray louder than radio-quiet quasars at high redshifts (Brinkmann et al. 1997) as well as at low redshifts (Zamorani et al. 1981). This is supported by our data, suggesting that an additional component contributes to the X-ray emission in radio-loud quasars and that radio-quiet and radio-loud quasars have distinct physical emission mechanisms. However the "X-ray quietness" of radio-quiet quasars makes a detailed spectral analysis rather difficult, and the small number of well studied radio-quiet high- $z$ quasars make definite conclusions rather uncertain.

No excess absorption has been found in the radio-quiet objects confirming that this property is common only in the high redshift, radio-loud quasars, even if not ubiquitous. A larger sample of high redshift radio-quiet quasars is needed to study this issue properly. As already mentioned for PKS 2126-158, damped Ly $\alpha$ systems have been discussed as possible X-rays absorbers for radio-loud high redshift quasars. Since its discovery Q 0000-263 is known to have a damped Ly $\alpha$ system lying along its line of sight at $z=3.39$ with $N_{\mathrm{HI}} \sim 2.6 \times 10^{21} \mathrm{~cm}^{-2}$ (Levshakov et al. 2000). We added the above fixed amount of absorbing material at the $\operatorname{Ly} \alpha$ system's redshift to the power law fit of Q 0000-263 and could not find any statistically significant differences in the fit parameters from a fit with galactic absorption only. The main differences in the two models occur at lowest energies $(\leq 0.3 \mathrm{keV})$ where the $\mathrm{PN}$ is not sensitive enough. As the count rates for Q 0000-263 are quite low, especially for the RGS, this means that the data are insufficient to determine the amount of absorbing material at high redshift for this source.

\section{Summary}

A brief summary of source properties and results of the spectral analyses are given in Table 7. Please note that we give, for a comparison, the power law slope for the PN fit with free absorption; the resulting $\chi_{\text {red }}^{2}$, given in the last column, indicates the quality of the fit. A simple power law fit for PKS 2149-306 with $\Gamma \sim 1.53$ and $N_{\mathrm{H}}=2.94 \times 10^{20} \mathrm{~cm}^{-2}$, slightly in excess of the galactic value, provides an acceptable description of the data in the $0.2-10 \mathrm{keV}$ energy band. Allowing for extra cold or warm absorption at the redshift of the source results in equally acceptable fits with an identical slope and $N_{\mathrm{H}, z} \sim 10^{21} \mathrm{~cm}^{-2}$. However the $N_{\mathrm{H}, z}$ is ill-determined and other redshifts of the absorber are compatible with the data. With the high signal to noise ratio from the EPIC data there is evidence for substantial deviations from a simple power law, such as a slightly curved shape of the residuals at soft energies, which can be modeled as a black body component with $k T \sim 0.25 \mathrm{keV}$, different power law slopes when different energy ranges are used for the fits, and the flatter slopes found from the MOS fit. We conclude that there is no strong evidence for extra absorption for this quasar. The addition of a narrow line at $\sim 5 \mathrm{keV}$ as claimed by Yaqoob et al. (1995) with a rest frame equivalent width of $\sim 12 \mathrm{eV}$ is not significant. Only a further improved signal to noise ratio would allow to separate different components in the spectrum.

For PKS 2126-158 extra absorption was found with $N_{\mathrm{H}}=$ $1.1 \times 10^{21} \mathrm{~cm}^{-2}$ and a power law slope of $\Gamma \sim 1.51$. In fits with extra absorption in the source frame, either neutral or through a warm absorber, values of $N_{\mathrm{H}, z} \sim 10^{22} \mathrm{~cm}^{-2}$ and $\Gamma \sim 1.47$ were found. However the redshift of the absorbing medium is poorly constrained. Further, deviations from the simple power law which cannot be modeled properly due to the still insufficient number of detected photons, seem to be present. A higher signal to noise ratio is needed, both to study the presence of the various spectral components and to determine the redshift of the absorber.

Both radio-loud quasars have high $2-10 \mathrm{keV}$ luminosities of the order of $\sim 10^{47} \mathrm{erg} \mathrm{s}^{-1}$ and they are X-ray loud with $\alpha_{\text {ox }} \sim 1$. The X-ray emission of PKS 2149-306 is dominated by a beamed, flat blazar-like component produced by inverse Compton scattering; the shape of the SED similar to that of a blazar confirms this view. 
PKS 2126-158 is a GPS source and the absorber we detect in X-rays might be the same as that assumed in the free-free absorption model for GPS sources (O' Dea 1998; Lister et al. 2002). From its spectral properties, PKS 2126-158 seems to have blazar-like characteristics similar to those of PKS 2149-306, apparently in contrast with being a GPS source, with a jet oriented at a large angle to the line of sight. However, some GPS sources exist which show signs of relativistic beaming as typically found in blazars (Lister et al. 2002).

For both radio-quiet objects a simple power law with $\Gamma \sim 2$ and galactic absorption gives a good description of the data. The power law slopes we found are consistent with typical values at low redshifts. No iron lines have been detected. The 2-10 keV luminosities are of the order of $\sim 10^{45} \mathrm{erg} \mathrm{s}^{-1}$, much lower than for their radio-loud counterparts. The $\alpha_{\text {ox }}$ of about $\sim 1.7$ are considerably larger than for the two radio-loud quasars. Being much X-ray weaker than radio-loud quasars, the low signal to noise ratios inhibit the detection of possible deviations from a simple power law slope.

The two radio-loud objects are found in the upper - left region of the $\alpha_{\text {ro }}-\alpha_{\text {ox }}$ diagram, slightly offset from the average of the low $-z$ radio-loud quasars, similar to the $z>4$ quasars of Fabian et al. (1999). They are thus X-ray brighter than their low- $z$ counterparts and follow the $\alpha_{\mathrm{ox}}-l_{\mathrm{o}}$ relation of radio-loud quasars (Brinkmann et al. 1997). The two radio-quiet objects are significantly more X-ray quiet than those at low redshifts and their $\alpha_{\mathrm{ox}}$ and their spectral power law slopes are nicely in line with the redshift dependence of these objects (Bechtold et al. 2002).

Overall, our sources follow the general trends observed for other high- $z$ quasars. However, in contrast to previous work, the spectral parameters could be determined with much higher accuracy. The great advantage of the XMM-Newton instruments for studies of high redshift quasars is the large sensitivity of the instrument. This allows not only the detection but even a spectral study of these objects and, in the case of more luminous sources, to study small spectral deviations from a simple power law which are indicators of the physical conditions governing the emission of radiation over the wide energy band accessible in high- $z$ objects.

Acknowledgements. This research has made use of the NASA/IPAC Extragalactic Data Base (NED) which is operated by the Jet Propulsion Laboratory, California Institute of Technology, under contract with the National Aeronautics and Space Administration. This work is based on observations with XMM-Newton, an ESA science mission with instruments and contributions directly funded by ESA Member States and the USA (NASA).

\section{References}

Avni, Y., \& Tananbaum, H. 1982, ApJ, 262, L17

Avni, Y., \& Tananbaum, H. 1986, ApJ, 305, 83

Ballantyne, D. R., Iwasawa, K., \& Fabian, A. C. 2001, MNRAS, 323, 506

Bechtold, J., Elvis, M., Fiore, F., et al. 1994a, AJ, 108, 374

Bechtold, J., Siemiginowska, A., Shields, J., et al. 2002, Proc. of the meeting "Active Galactic Nuclei: from Central Engine to Host Galaxy"
Brinkmann, W., Siebert, J., Reich, W., et al. 1995, AAS, 109, 147

Brinkmann, W., Yuan, W., \& Siebert, J. 1997, A\&A, 319, 413

Boyle, B. J., Griffiths, R. E., Shanks, T., Stewart, G. C., \& Georgantopoulos, I. 1993, MNRAS, 260, 49

Cappi, M., Matsuoka, M., Comastri, A., et al. 1997, ApJ, 478, 492

de Vries, W. H., O’Dea, C. P., Baum, S. A., \& Barthel, P. D. 1997, A\&AS, 191.2204D

D’Odorico, V., Christiani, S., D'Odorico, S., Fontana, A., \& Giallongo, E. 1998, A\&AS, 127, 217

Ehle, M., Breitfellner, M., Dahlem, M., et al. 2001, XMM-Newton Users' Handbook,

http://xmm.vilspa.esa.es/xmm_user_support/ external/documentation/uhb_frame.shtml

Elvis, M., Wilkes, B. J., McDowell, J. C., et al. 1994a, ApJS, 95, 1

Elvis, M., Fiore, F., Wilkes, B. J., McDowell, J., \& Bechtold, J. 1994b, ApJ, 422, 60

Elvis, M., Fiore, F., Siemiginowska, A., Bechtold, J., Mathur, S., et al. 2000, ApJ, 543, 545

Fabian, A. C. 1979, Proc. R. Soc. London, Ser. A, 366, 449

Fabian, A. C., Celotti, A., \& Pooley, G. 1999, MNRAS, 308, L6

Fang, T., \& Canizares, C. 2000, ApJ, 532, 539

Fang, T., Marshall, H. L., Bryan, G. L., \& Canizares, C. 2001, ApJ, 555,536

Ghizzardi, S., \& Molendi, S. Proc. of the conference "New Visions of the X-ray Universe", ESTEC Nov. 2001

Giallongo, E., Cristiani, S., Fontana, A., \& Trevese, D. 1993, ApJ, 416, 137

Kaspi, S., Brandt, W. N., \& Schneider, D. P. 2000, AJ, 119, 2031

Lister, M. L., Kellermann, K. I., \& Pauliny-Toth, I. I. K. 2002, Proc. of the 6th European VLBI Network Symposium on New Developments in VLBI Science and Technology

Levshakov, S. A., Molaro, P., Centurion, M., et al. 2000, A\&A, 361, 803

Mushotzky, R. F., Done, C., \& Pounds, K. A. 1993, ARA\&A, 31, 717

O’Dea, C. P. 1998, PASP, 110, 493

Padovani, P., Costamante, L., Ghisellini, G., Giommi, P., \& Perlman, E. 2002, ApJ, 581, 895

Reimers, D., Bade, N., Schartel, N., et al. 1995, A\&A, 296, L49

Reeves, J. N., \& Turner, M. J. L. 2000, MNRAS, 316, 234

Sanduleak, N., \& Pesch, P. 1989, PASP, 101, 1081

Schartel, N., Walter, R., Fink, H. H., \& Trümper, J. 1996, A\&A, 307, 33

Schartel, N., Komossa, S., Brinkmann, W., et al. 1997, A\&A, 320, 421

Serlemitsos, P., Yaqoob, T., Ricker, G., et al. 1994, PASJ, 46, L43

Siebert, J., Matsuoka, M., Brinkmann, W., et al. 1996, A\&A, 307, 8

Sikora, M., Madejski, G., Moderski, R., \& Poutanen, J. 1997, ApJ, 484, 108

Stanghellini, C., O’Dea, C. P., Dallacasa, D., et al. 1998, A\&AS, 131, 303

Wilkes, B. J., \& Elvis, M. 1987, ApJ, 323, 243

Wilkes, B. J., Tananbaum, H., Worrall, D .M., Avni, et al. 1994, ApJS, 92, 53

Worrall, D. M., \& Wilkes, B. J. 1990, ApJ, 360, 396

Yaqoob, T., George, I. M., Nandra, K., et al. 1999, ApJ, 525, L9

Yuan, W., Brinkmann, W., Siebert, J., \& Voges, W. 1998a, A\&A, 330, 108

Yuan, W., \& Brinkmann, W. 1998, in Highlights in X-ray Astronomy, ed. B. Aschenbach, \& M. J. Freyberg, MPE Report, 272, 240

Yuan, W., Siebert, J., \& Brinkmann, W. 1998b, A\&A, 334, 498

Yuan, W., Matsuoka, M., Wang, T., Ueno, S., Kubo, H., et al. 2000 , ApJ, 545, 625

Zamorani, G., Henry, J. P., Maccacaro, T., et al. 1981, ApJ, 245, 357 